Rhode Island College

Digital Commons @ RIC

2015

\title{
An Evaluation of the Palliative Care Provided on a Medical Intensive Care Unit
}

Peter Musso

Rhode Island College

Follow this and additional works at: https://digitalcommons.ric.edu/etd

Part of the Nursing Commons

\section{Recommended Citation}

Musso, Peter, "An Evaluation of the Palliative Care Provided on a Medical Intensive Care Unit" (2015).

Master's Theses, Dissertations, Graduate Research and Major Papers Overview. 160.

https://digitalcommons.ric.edu/etd/160

This Major Paper is brought to you for free and open access by the Master's Theses, Dissertations, Graduate Research and Major Papers at Digital Commons @ RIC. It has been accepted for inclusion in Master's Theses, Dissertations, Graduate Research and Major Papers Overview by an authorized administrator of Digital Commons @ RIC. For more information, please contact digitalcommons@ric.edu. 


\title{
AN EVALUATION OF THE PALLIATIVE CARE PROVIDED ON A MEDICAL INTENSIVE CARE UNIT
}

\author{
by \\ Peter Musso \\ A Major Paper Submitted in Partial Fulfillment \\ of the Requirements for the Degree of \\ Master of Science in Nursing \\ in \\ The School of Nursing \\ Rhode Island College
}

2015 


\begin{abstract}
\end{abstract}
More patients die in intensive care units (ICUs) than in any other hospital setting. For survivors, ICU treatment is often accompanied by a significant burden of symptoms for both the patient and for the family and may result in long-term cognitive and physical impairments and an unacceptable quality of life. Over the last decade, the idea that palliative care should be provided along with intensive care regardless of prognosis has evolved from a novel formulation to a clinical practice guideline. The purpose of this research was to determine whether the patients on a Medical Intensive Care Unit (MICU) were being offered appropriate palliative care. A retrospective chart review of 250 charts was performed at a 653-bed acute care, teaching facility located in southern New England with a sample of 50 patients. The Care and Communication Bundle was used to measure if primary palliative care needs were met; the Palliative Care Service Consult Tool developed by the palliative care team at the study institution was used to measure if tertiary palliative care needs were met. Results indicated approximately $85 \%$ compliance with primary palliative care overall, compliance with the individual items ranged from $40 \%-100 \%$. Tertiary palliative care compliance was $7 \%$ overall, with only two out of 29 patients actually receiving a consult. Recommendations for practice change include integrating the tool into the electronic medical record as part of the admission assessment. Interdisciplinary staff education on the process and use of the measures is indicated. State and national policies related to palliative care would facilitate the implementation of palliative care programs aimed at providing care for all people in need of these services and ensure equitable access to end-of-life care. Advanced practice nurses have a key role 
in advocating for policy changes within their institutions, as well as, at the state and national levels that could help patients meet their goals of care, especially at the end of their lives.

Keywords: palliative care, hospice, intensive care, standards, and guidelines 


\section{Table of Contents}

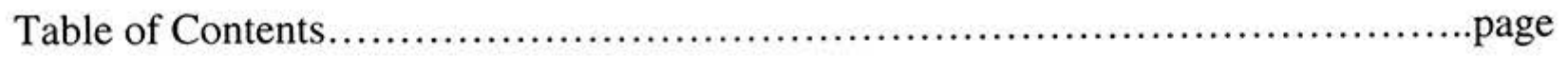

Background and Statement of the Problem.........................................

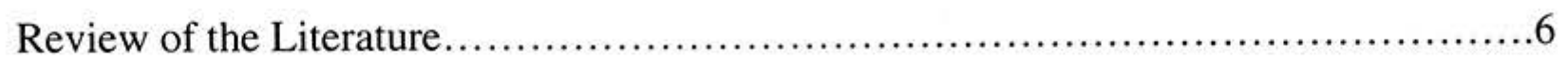

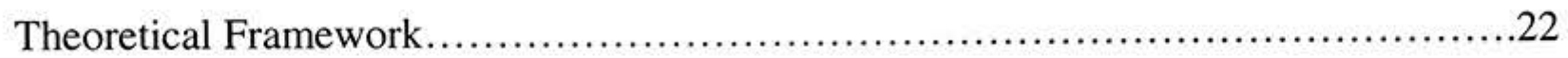

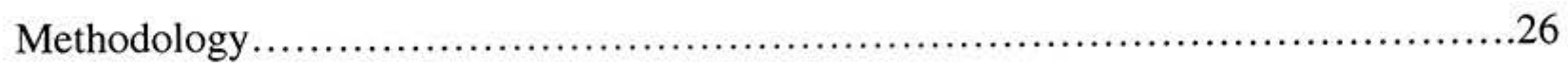

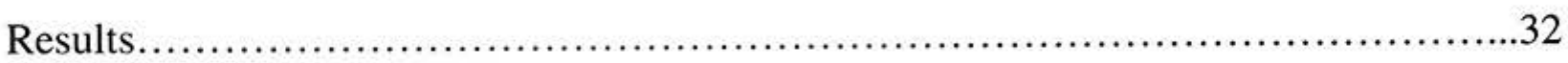

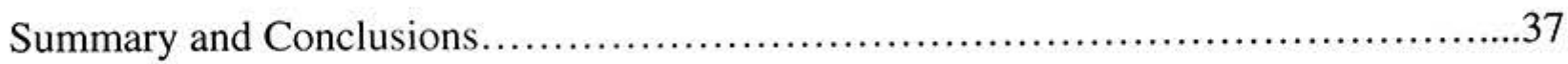

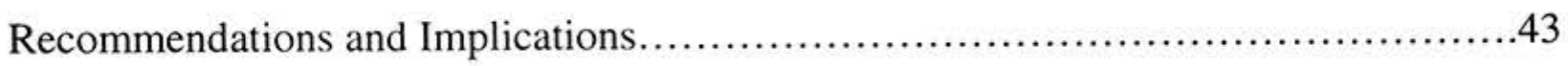

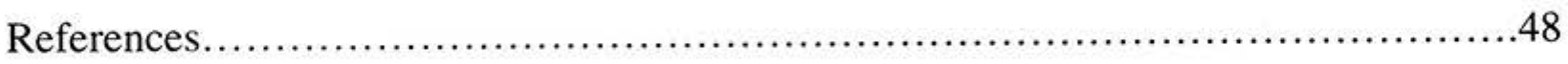

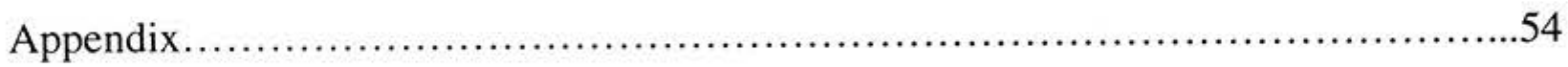


An Evaluation of the Palliative Care Provided on a Medical Intensive Care Unit

\section{Background and Statement of the Problem}

More patients die in intensive care units (ICUs) than in any other hospital setting. For survivors, ICU treatment is often accompanied by a significant burden of symptoms for both the patient and for the family, and may result in long-term cognitive and physical impairments with an unacceptable quality of life (Nelson et al., 2010). Before high-tech ICUs and modern medicine, seriously ill people died at home, surrounded by their loved ones, who clearly knew best how to succor and comfort family members as death approached. The ability to be an intimate part of the dying process with a loved one allowed family members to feel as if they were "doing everything" or providing the best care possible. Unfortunately, as medical science has progressed, the definition of "doing everything" for a loved one has changed (Levy, 2001). The atmosphere of the healthcare world a decade ago was an awakening to the void in the care of the critical ill patients at the end of their lives.

Critical illness once was distinguished clearly from 'terminal illness'. Patients were brought to ICUs to save their lives, not to provide end-of-life care. For most patients, the discussion about comfort and end of life goals moved to the center of attention only after hope of cure or substantial prolongation of life was lost (Nelson \& Danis, 2001). Palliative care was postponed until death was known to be near.

The concept of palliative care evolved from the hospice philosophy of filling the gaps in care for seriously ill and dying patients. The Latin word palliate means "conceal or alleviate symptoms without curing" (Morgan, 2009, p. 86). Hospice can be traced to 
religious orders during the Middle Ages, when they established 'hospices' at key crossroads on the routes to religious shrines. Many pilgrims travelled these routes to the shrines seeking miraculous cures of chronic and fatal illnesses; many died while on the pilgrimages. The word 'hospice' comes from the Latin word 'hospes' meaning to host a guest or stranger (Amitabha Hospice Service, 2009). During the $16^{\text {th }}-18^{\text {th }}$ centuries, the religious orders offered care to the sick and dying in local and regional-based institutions. At that time, most people died at home while being cared for by the women in the family (Hospice Education Institute, 2002).

The name hospice was first applied to the care of dying patients by Madame Jeanne Garnier who founded the Dames de Calaire in Lyons, France in 1842. The name was next introduced by the Irish Sisters of Charity when they opened Our Lady's Hospice in Dublin in 1879 and St. Joseph's Hospice in Hackney, London in 1905. In 1967, Dame Cicely Saunders started St. Christopher's Hospice after being inspired by a patient, David Tasma, whom she met in 1948 when he was hospitalized with an inoperable cancer and she, a former nurse, was working as a medical social worker. The two discussed how she might one day open a place that was better suited to pain control and preparing for death than a busy hospital ward. When he died, he bequeathed her some money and told Saunders, "I will be a window in your home." Since then her ideas have spread around the world, which gave her the reputation of being the founder of the modern hospice movement (Amitabha Hospice Service, 2009).

In 1969, a book based on more than 500 interviews with dying patients was published, written by Dr. Elizabeth Kubler-Ross. The book, On Death and Dying, 
became a best seller and took death out of secrecy and into public awareness and discussion for the first time. Dr. Kubler-Ross argued that home care was preferable over institutional care and that patients should be able to participate in decisions regarding their treatment (Amitabha Hospice Service, 2009). She emphasized patients' values, preferences and goals which are an important component of today's palliative care.

The year 1974 saw the first hospice home care in the United States (US), when New Haven Hospice, now Connecticut Hospice, was established and started home care services for people with cancer, ALS and other fatal illnesses. The first US hospital-based palliative care programs began in the late 1980's at a handful of institutions such as the Cleveland Clinic and Medical College of Wisconsin. The term 'hospice palliative care' was coined to recognize the convergence of hospice and palliative care into one movement and their common norms of practice. While hospice palliative care is the nationally accepted term to describe care aimed at relieving suffering and improving quality of life, individual organizations may continue to use 'hospice', 'palliative care', or another similarly acceptable term to describe their organization and the services they are providing (North Simcoe Muskoka Palliative Care Network, 2012). In the summer of 2001, the Institute of Medicine (IOM), in partnership with the National Research Council, recognized the need for improvement of end-of-life and palliative care in their report, Improving Palliative Care in Cancer (Foley \& Gelband, 2001). Despite major advances in the management of some cancers, $50 \%$ of all patients being diagnosed with cancer were dying within a few years. Dying from cancer has become synonymous with a spectrum of symptoms, including pain, labored breathing, distress, nausea, confusion, 
other physical and psychological conditions, that go untreated or undertreated and vastly diminish the quality of those lives (Chertkov et al., 2001). The authors reported many issues that are relevant across the globe and highlighted some of the damaging anomalies within the American healthcare system. The report cited no villains apart from ourselves and our culture. The two main deficiencies identified in the report were symptom management of the critically ill and effective communication about goals of care between clinicians, patients and families.

There have been many reports and studies since the IOM's report citing the deficiencies in our end-of-life care of the critically ill patients. One such report was Describing Death in America, What We Need to Know by The National Cancer Policy Board (Lunney, Foley, Smith, \& Gelband, 2003). The authors identified that there was insufficient information to assess the quality of care that was provided to those who died from cancer in the United States. This lack of information hampered their ability to develop a clear policy agenda and impeded monitoring trends to determine whether interventions were having the intended effects of improving the quality of life and care for individuals at the end of life. 'Quality of care' is a subjective concept, but at the time of the report, various groups had begun to define minimum standards that could be agreed upon, as well as ideas that could be held as goals. Quality of care is not an end in itself, either for the temporarily or the fatally ill. It is one contributor to 'quality of life,' regardless of the amount of time left in a life. In this report, Lunney et al. were concerned with describing both the 'quality of care' and 'quality of life' near the end of life. "They are distinct qualities and require different types of measurements, related either to the 
process and outcomes of care, in the former, or the perceptions of the dying and those around them, in the latter" (p. 16).

Over the last decade, the idea that palliative care should be provided along with intensive care regardless of prognosis has evolved from a novel formulation to a clinical practice guideline (Nelson et al., 2011). Palliative care is a dynamic field and is now recognized as a medical specialty. Expert palliative care through a consultation service is currently available at the majority of US hospitals, including greater than $75 \%$ of large hospitals, all Veterans' Affairs medical centers, and an increasing number of smaller and community-based hospitals (Nelson et al., 2010). Through the evolution of palliative care in the past decade, the Center to Advance Palliative Care (CAPC) has been a major player in the changes seen today. In 2010, CAPC and the National Institutes of Health (NIH) sponsored a project called Improving Palliative Care in the ICU (IPAL-ICU). The project highlighted the importance of the ICU as a venue for providing and improving palliative care, identified opportunities for improvement of ICU palliative care, and described expected benefits of an ICU-palliative care initiative. Noted were the present deficiencies in the ICU palliative care and the areas that clinicians need to address through the Care and Communication Bundle of nine process measures of quality established by the Agency for Healthcare Research and Quality (AHRQ).

The purpose of this research project was to evaluate whether the patients on a Medical Intensive Care Unit (MICU) were being offered appropriate palliative care, using established tools, the Care and Communication Bundle and the Palliative Care Service Tool. Next, the review of the literature will be presented. 


\section{Review of the Literature}

The databases searched included Cinahl Plus, Pub Med, and Ovid from April of 1998 to 2013, including all English language articles using the terms 'palliative care,' 'hospice,' 'intensive care,' 'standards,' and 'guidelines.'

\section{Contemporary Historical Perspective}

Palliative care was first introduced by the World Health Organization (WHO) in 1990, when the organization set standards for palliative care and pain control, identifying palliative care as a priority. Palliative care was assigned a higher priority, as witnessed by the construction of palliative care centers, where training modules, study groups, cancer and palliative care programs were developed. Noteworthy also was the recommendation to introduce such services in public national health care systems (Dumitrescu, 2006).

In 1997, the Institute of Medicine report, Approaching Death: Improving Care at the End of Life, documented glaring deficiencies in end-of-life care in the US. The IOM report stressed several themes that reflected the broad deficiencies that exist in the care of people with life-threatening, incurable illnesses as identified below.

The study sought to evaluate the state of knowledge in the field, methods for assessing outcomes, patients' preferences, and the quality of care and to identify barriers to high-quality care and propose steps for improvement. An impressive amount of thought and energy went into the work of the 12-member committee of experts and its staff, which held public meetings, reviewed and critiqued literature and testimony, and compiled a 418 page report. As a summary and critique of the 
state of affairs nationally, the IOM report can certainly be viewed as a definitive work. (Field and Cassel, 1998, p. 437)

Some of the deficiencies identified by Field and Cassel (1997) were: preventable pain and suffering was experienced too often by dying individuals; there were significant organizational, legal, and educational obstacles to good care; there were important gags in evidenced-based knowledge about end of life care; the education and training of physicians and other healthcare professionals fail to provide them with the knowledge and skills required to care for dying patients; and better data and tools were needed for evaluating the outcomes important to patients and families during end-of-life.

The IOM report contained several recommendations aimed at addressing and remedying the healthcare system's shortcomings. Because many problems in care stemmed from system problems, it was proposed that: the policymakers, consumer groups, and purchasers of healthcare should work with healthcare practitioners, organizations, and researchers to strengthen methods for measuring the quality of life and other outcomes of care for dying patients and their families; develop tools and strategies for improving the quality of care and holding healthcare organizations accountable for care at the end of life; revise mechanisms for financing care so that they encourage rather than impede good end-of-life care; and reform drug prescription laws and regulations, and state medical board policies and practices that impede effective use of opioids to relieve pain and suffering (Chertkov et al., 2001). From the stated deficiencies and recommenda-tions of the IOM (Field \& Cassel, 1997), crafters influencing the healthcare 
system had a foundation on which to build palliative care services.

\section{Evolution and Definition of Palliative Care}

The WHO (2004) defined palliative care as "an approach that improves the quality of life of patients and their families facing the problem associated with lifethreatening illness, through the prevention and relief of suffering by means of early identification and impeccable assessment and treatment of pain and other problems, physical, psychosocial and spiritual" (WHO, 2004, para. 1). The WHO definition affirms life and regards dying as a normal process, not intending to hasten or postpone death, supports both patient and family during the patient's illness to improve the quality of life, and to do all this in conjunction with other therapies that are intending to prolong life.

The Center to Advance Palliative Care (CAPC) provided another definition of palliative care: "a specialized medical care for people with serious illnesses. Palliative care focuses on providing patients with relief from symptoms, pain and stress of a serious illness - whatever the diagnosis. The goal is to improve quality of life for both patient and family" (CAPC, 2006, Defining Palliative Care, para. 1). The CAPC further identified specific serious and chronic illnesses where palliative care is used as a treatment to improve the quality of life. These illnesses include cancer, cardiac disease such as congestive heart failure (CHF), chronic obstructive pulmonary disease (COPD), kidney disease, Alzheimer's, HIV/AIDS and amyotrophic lateral sclerosis (ALS). The CAPC advocates working with the doctor to provide an extra layer of support. The palliative care team provides: time for close communication between clinicians and patients and their families; expert management of pain and other distressing symptoms; 
helps the patients to navigate the healthcare system; provides guidance with difficult and complex treatment choices; and offers emotional and spiritual support to patients and their families (CAPC, 2006).

The CAPC provides health care professionals with the tools, training, and technical assistance necessary to increase access to quality palliative care services in hospitals and other health care settings (CAPC, 2011). The CAPC has become a major force in the field of palliative care due to the increase demand for training and support. Over the last ten years, palliative care has been one of the fastest growing trends in health care. The number of palliative care teams within hospitals has increased $148 \%$, from more than 600 in 2000 to more than 1600 in 2012 (CAPC, 2012).

There are many similarities between these two definitions that are the foundation of the palliative care services of the US healthcare system. These include improving the quality of life for both the patient and the family, relief from distressing symptoms, emotional and spiritual support for both the patient and the family, working in conjunction with the present therapy, and enhancing the communication between clinicians, patients, and families.

\section{Clinical Practice Guidelines for Quality Palliative Care}

In 2004, the National Consensus Project developed the first edition of the Clinical Practice Guidelines for Quality Palliative Care (Appendix A), identifying eight important domains in the creation and maintenance of quality palliative care. Within each domain, there are guidelines that define optimal practice. The guidelines start with the principles of assessment, information sharing, decision-making, care planning, and care 
delivery. Within the guidelines for each domain, there are corresponding descriptions, clarifying statements and assessment criteria for meeting the expectation.

The underlying tenets of palliative care in the document include: patient and family centered palliative care; comprehensive palliative care with continuity across health settings; early introduction of palliative care at diagnosis of a serious disease or life threatening condition; interdisciplinary collaborative palliative care; clinical and communication expertise within palliative care team members; relief of physical, psychological, emotional, and spiritual suffering and distress of patients and families; a focus on quality; and equitable access to palliative care services (National Consensus Project, 2013).

\section{Bundling Palliative Care}

Still in its infancy, palliative care deficiencies have been well documented in the literature in the past two decades. As the population ages and intensive care treatments are offered to older and sicker patients, the quality of end-of-life for patients in the ICUs will be ever more important. Healthcare continues to confront major barriers to better care, some arising from deep in the culture of ICU medicine (Nelson, 2006).

Over the past decade, clinicians have become familiar with the bundle approach to care in which combined evidenced-based processes are applied together to improve quality of care. Two widely successful bundles have included those for the prevention of ventilator-associated pneumonia and catheter-related bloodstream infection (Nelson et al., 2010). To address the deficiencies of palliative care being delivered in intensive care units (ICUs), the Voluntary Hospital Association (VHA) Inc. sponsored the development 
of the Care and Communication Bundle (Appendix B) of care process measures of quality as part of its Transformation of the ICU (2006) performance improvement initiative. Through its Transformation of the ICU project, VHA has assisted more than 75 ICUs to implement 'bundles' of quality measures for sepsis, mechanical ventilation, and catheter-related bloodstream infection, and it developed a new palliative care bundle to improve comfort and communication for critically ill patients and their families (Agency for Healthcare Research and Quality [AHRQ], 2006).

\section{Palliative Care Needs Identified by Patients and Their Families}

Despite considerable technologic breakthroughs in the provision of intensive care during the last 40 years, mortality in the ICU remains high, ranging from $6.4 \%$ to $40 \%$ across ICUs, depending on the severity of illness (Angus et al., 2004). Of those patients that leave the ICU, 100,000 ICU 'survivors' continue with critical illness on a chronic basis. In a report published by the CAPC in 2010, The Improving Palliative Care in the ICU (IPAL-ICU) project, 20\% of Americans, or about 500,000 people per year, die in or shortly after an intensive care stay. The majority of these deaths are preceded by a decision to withhold or withdraw life-sustaining therapies. A growing body of evidence, to be reviewed next, has also pointed out the dissatisfaction expressed by the families with the quality of care received by their loved ones who died while in the intensive care unit.

The Study to Understand Prognoses and Preferences for Outcomes and Risks of Treatment (SUPPORT) was a two phase, controlled trial to improve care for seriously ill hospitalized patients (Connors et al., 1995). The objective of the trial was to improve 
end-of-life decision making and reduce the frequency of a mechanically supported, painful, and prolonged process of dying. Phase I was a prospective observational study that described the process of decision making and patient outcomes. In phase I, the researchers documented the shortcomings in communication, frequency of aggressive treatment, and the characteristics of hospital death. During phase II, the physicians in the intervention group received estimates of the likelihood of six month survival, outcomes of cardiopulmonary resuscitation (CPR), and functional disability at two months. Specially trained nurses had multiple contacts with the patient, family, physician, and hospital staff to elicit preferences, improve understanding of outcomes, encourage attention to pain control and facilitate advance care planning and patient-physician communication. During the phase II intervention, patients experienced no improvement in patient-physician communication or in the five targeted outcomes. These included incidence or timing of written do-not-resuscitate (DNR) orders, physicians knowledge of their patients' preferences not to be resuscitated, number of days spent in an ICU, receiving mechanical ventilation, comatose before death, and level of reported pain. The authors concluded that to improve the experience of the seriously ill and dying patients, greater individual and societal commitment and more proactive and forceful measures may be needed.

In a qualitative study, Nelson et al. (2010) randomly selected patients with intensive care unit length of stay greater than five days who survived the intensive care unit, families of survivors, and families of patients who died in the intensive care unit were placed in focus groups and interviewed. Although the majority of hospital deaths 
occur in the ICUs, and all critically ill patients and their families have palliative care needs, little research had been done to identify how patients and families, the most important stakeholders, define high-quality palliative care. The purpose of the study was to obtain their views on important domains of this care. There were a total of 1,240 participants who were separated into three focus groups: the patients; family members of patients who survived the ICU; and family members of patients who died in the ICU. The focus group participants identified four aspects of ICU palliative care that were most important to them. These included communication by clinicians about the patients' condition, treatment and prognosis; patient-focused medical decision-making, clinical care of the patient to maintain comfort, dignity, personhood, and privacy and care of the family. The authors noted that critical care professionals have committed energy and resources to improve quality and safety in every major area of their practice, from ventilator management to prevention of catheter-related bloodstream infections. They emphasized that it is essential that such efforts focus on aspects of palliative care that are most valued by critically ill patients and their families, among whom they found broad agreement in their sample from heterogeneous institutions.

High quality care for ICU patients and their families includes palliative care. To promote performance improvement, the AHRQ National Quality Measures Clearinghouse identified nine evidence-based processes of intensive care unit palliative care in the form of the Care and Communication Bundle (Appendix B) through a review of medical record documentation (Penrod et al., 2012). Penrod et al. conducted a prospective, multisite, observational study to examine how frequently the Care and 
Communication Bundle processes were performed in three diverse ICUs across the country and to understand patient factors associated with such performance. The authors found that the performance of the evidence-based process measures of high quality palliative care were inconsistent and infrequent. For example, interdisciplinary family meetings to discuss goals of care in relation to the patients' condition, prognosis, and preferences were documented for $<20 \%$ of patients, and occurred as late as five days after admission to the ICU for critical care treatment. Information in printed form was rarely distributed to families, despite evidence from an earlier randomized, controlled, multicentered trial (Azoulay et al., 2002) that demonstrated the effectiveness of this low-cost approach. Azoulay et al. performed this prospective trial in 34 French ICUs to compare comprehension of diagnosis, prognosis, treatment, and satisfaction with information provided by ICU caregivers. They compared those family members who did receive a family information leaflet (FIL) and those who did not. Use of the FIL reduced the proportion of family members with poor comprehension from $40.9 \%$ to $11.5 \%$. Penrod et al. (2012) concluded that methods used to improve the quality and safety of other aspects of ICU care, including emphasis on efficient work systems, practical tools and interdisciplinary teamwork show promise for ensuring delivery of high-quality palliative care in the ICU. Palliative care and end-of-life care is changing in the US.

This dynamic field of palliative care is improving the care for patients with serious and life-threatening cancer through creation of national guidelines for quality care (Appendix A), multidisciplinary educational offerings, research endeavors, and resources made available to clinicians. Barriers to implementing quality palliative care across 
cancer populations include a rapidly expanding population of older adults who will need cancer care and a decrease in the workforce available to give care. One approach in addressing the workforce shortage is the recognition of palliative care as a medical specialty. An important component is the increased use of palliative care physicians and nurse practitioners in meeting many of these unmet needs, as well as the coordination of the different medical specialties that are involved in the patient's care (Grant, Elk, Ferrell, Morison, \& Von Gunten, 2009). Grant et al. presented the current status of palliative care, the challenges to implementing palliative care, methods to improve application of palliative care, and the clinical implications for clinicians involved in caring for patients with advanced cancer.

Communication about patient goals and preferences for care is another aspect of care that was often lacking (Grant et al., 2009). When serious and life-threatening illness occurred, the patients voiced their priorities as pain and symptom control, avoidance of prolongation of the dying process, a sense of control, and an opportunity to strengthen relationships with loved ones. Grant et al. commented that research does not demonstrate that patients' preferences are adequately met. The author reported that moderate to severe pain was documented by $60 \%$ of patients with colon cancer and $57 \%$ of patients with lung cancer. On average, it took between eight and 14 days to get pain under control. Family members also reported poor emotional support, a lack of respectful treatment, and no involvement in decision making about care. As healthcare professionals, clinicians are responsible to learn more about palliative care to overcome some of these barriers. Clinicians cannot practice what they do not know; attending presentations about 
palliative care to increase their knowledge base is an essential initial step (Grant et al.). The authors concluded that multidisciplinary educational initiatives, clerical applications, and research studies have begun to move palliative and end-of-life toward the recommendations identified in the National Consensus Guidelines (Appendix A).

\section{Palliative Care in the Intensive Care Unit}

Campbell and Guzman (2003) compared patterns of end-of-life care for patients with multiple organ system failure (MOSF) and global cerebral ischemia (GCI) after cardiopulmonary resuscitation. The authors conducted a comparative study of retrospective and prospective cohorts to assess the impact of a proactive case finding approach to end-of-life care. Patterns of end-of-life care for these patients obtained through a retrospective chart review were compared to a proactive case finding facilitated by the inpatient palliative care service. In the retrospective review, 404 medical records were identified; 40 patients met the inclusion criteria, 18 patients with GCI and 22 patients with MOSF. The proactive cohort consisted of 20 patients with GCI and 21 patients with MOSF that met the inclusion criteria. The authors demonstrated a considerable time lag elapsed between identification of poor prognosis and the establishment of end-of-life treatment goals in the retrospective patients $(4.7 \pm 2.4$ days and $3.5 \pm 0.5$ days for patients with MOSF and GCI, respectively). At the time of admission, all the patients in the proactive cohort, both subgroups of GCI and MOSF, had a DNR order in comparison to $88 \%$ of patients with GCI and $91 \&$ of patients with MOSF in the retrospective group. Comfort measures were chosen twice more frequently than withholding resuscitation during the proactive trial. The authors also illustrated that the 
proactive palliative care intervention decreased length of stay in the hospital $(20.6 \pm 4.1$ days vs. $15.1 \pm 2.5$ days and $8.6 \pm 1.6$ days vs. $4.7 \pm 0.6$ days for MOSF and GCI patients, respectively), decreased the time dying patients with MOSF remained in the ICU, and reduced the use of non-beneficial resources, thus reducing the cost of care.

Nelson et al. (2010) carried out a literature review of MEDLINE database from inception to April 2010 using the terms 'intensive care' and 'palliative care'. The authors reviewed the existing data with a focus on models that have been used to structure clinical initiatives to enhance palliative care for critically patients in the ICU and their families. Palliative care focuses on complex pain and symptom management, communication about care goals, alignment of treatments with the patient values and preferences, transitional planning. and support for the family. This type of care is seen as an essential component of comprehensive care for patients with critical illness, including those receiving aggressive intensive care. The authors commented that although prior literature has illustrated why palliative care could be improved, guidance on how this might be effectively accomplished in practice remains limited. This article focused on practical approaches to ICU palliative care and introduced two main models for ICUpalliative care integration: (1) the "consultative model," which utilizes the medical specialty of a palliative care team in the care of the critically ill intensive care patients and their families; and (2) the "integrative model," which seeks to root the principles of palliative care into daily practice of the intensive care team for all patients and families facing critical illness. Nelson et al. described the key features of both models, which could be used alone or in combination. The authors also discussed their advantages and 
disadvantages, provided examples of initiatives using different models, addressed the process of choosing an appropriate model, and reviewed outcomes of effective integration of palliative care in the ICU setting.

All ICU patients and their families have palliative care needs and Nelson et al. (2010) noted that many critical care professionals believe that the ICU team should integrate palliative care principles into their daily ICU practice. This approach requires internal efforts to enhance systems of care and ICU clinicians' knowledge and skill in palliative care. Thus, educational efforts targeted to physicians, nurses, and other members of the critical care team are a key component of initiatives to strengthen the internal capability for ICU palliative care. Nelson et al. commented that whichever model that is utilized, it is only a well-structured initiative that will enhance the palliative care in the ICU, provide important benefits for patients, families, and providers, and be associated with reductions in the use of non-beneficial ICU treatments, length of stay, and/or conflict over care goals. The primary mechanism for efficiencies in use is earlier clarification of patients' preferences, consensus in decision making, and allowing timely implementation of an appropriate plan of care (Nelson et al.).

Patients diagnosed with metastatic non-small-cell lung cancer have a significant symptom burden, and often receive aggressive care at the end of their life. Temel et al. (2010) examined the effect of introducing palliative care early after diagnosis on patientreported outcomes and end-of-life care among ambulatory patients with newly diagnosed disease. The authors conducted a randomized controlled study of 151 patients with newly diagnosed metastatic non-small-cell lung cancer. The patients were randomly assigned to 
either receive early integration of palliative care in addition to standard oncologic care or oncologic care alone. Patients in the palliative care group had at least one palliative care visit per month until death. The patients were assessed at baseline and at 12 weeks using the Functional Assessment of Cancer Therapy-Lung (FACT-L) scale and the Hospital Anxiety and Depression Scale. The authors demonstrated that early palliative care lead to significant improvements in both quality of life (mean score on the FACT-L scale [in which scores range from 0-136, with higher scores indicating better quality of life] 98.0 vs. $91.5 ; \mathrm{P}=0.03$ ). Fewer patients in the palliative care group than in the standard care group had depressive symptoms ( $16 \%$ vs. $38 \%, p=0.01$ ). As compared with patients receiving standard care, patients receiving early palliative care had greater documentation of resuscitation preferences and less aggressive care at the end of life and longer survival (11.6 months vs. 8.9 months, $\mathrm{p}=0.02$ ).

The label of receiving "prolonged mechanical ventilation" has been placed on 300,000 patients per year who receive life support in the ICUs for much longer than the average patient. These cases utilize a disproportionately large amount of healthcare resources and have relatively poor long-term outcomes. Unroe et al. (2010) performed a one-year prospective cohort study, enrolling 126 patients who underwent prolonged mechanical ventilation. The authors then followed them and their surrogates for 12 months. Prolonged ventilation was defined as ventilation for $\geq$ four days with tracheostomy placement or ventilation for $\geq 21$ days without tracheostomy placement. The focus of the study was quality of life and healthcare utilization after their initial hospitalization. The authors found that these patients used a disproportionately large 
amount of health care resources and have relatively poor long-term outcomes. Poor outcomes, as reported by patients, include diminished quality of life, functional and cognitive limitations requiring prolonged informal caregiver assistance. Participants also had a high one-year mortality rate. Quality of life was defined by the EuroQol-5D (EQ5D), an instrument with established validity in survivors of critical illness. Surrogates completed proxy measure. Scores on the EQ-5D were highly correlated $(r=0.94$; $\mathrm{P}<0.001)$ between surrogates and cognitively intact patients. At the one year mark, 70 patients (56\%) were alive, although only $11(9 \%)$ were independently functioning and only $19(27 \%)$ had a 'good' quality of life. There were 150 readmissions in $68(67 \%)$ of the 103 hospital survivors. Most readmissions occurred within three months; nearly half were related to sepsis. The average patient spent $74 \%$ of all days alive in a hospital or post-acute care facility or receiving home health care. Only three patients $(2 \%)$ were both initially discharged to their home and remained there, whereas only three of fifty-four previously employed patients ever returned to work. The mean cost of care per patient over the year was $\$ 306,135$, amounting to a $\$ 3.5$ million cost for each independently functioning survivor (Unroe et al.). This study confirms that prolonged mechanical ventilation is a highly resource-intensive condition with generally poor outcome.

\section{Critique of the Literature}

Approximately $20 \%$ of all deaths in the US occur in the ICU or shortly after a stay in the ICU, making the link between intensive care and palliative care that much stronger. All critical ill patients and their families have palliative care needs that need to be identified and met. As the literature continues to demonstrate, there are still deficits in the 
palliative care delivered: many people continue to die in moderate to severe pain; physicians are frequently unaware of their patients' preferences regarding end-of-life care and resuscitation preferences; family caregiver needs for spiritual and social work support remain unmet; and open communication among patients, families, and health care providers concerning the plan and goals of care is frequently lacking.

Tools are available to provide critical ill patients with the palliative care they need, and through the use of the Care and Communication Bundle (Appendix B) it is possible to systematically evaluate if there needs are being met. Treatment needs to move from a disease-focused approach to a patient-centered philosophy, where the needs of the patient and the patient/family goals are essential to planning care. Initiating early discussion about patient preferences and palliative needs and structured daily reassessment is an approach that promotes excellent palliative care simultaneously with curative and life-sustaining therapies in the ICU. The purpose of this study was to determine whether patients on a MICU were offered appropriate palliative care.

Next, the theoretical frameworks used to guide this research will be presented and discussed. 


\section{Theoretical Framework}

This study design was guided by the Theory of Peaceful End of Life (Rulund \& Moore, 1998) and by the Framework for Program Evaluation (Centers for Disease Control [CDC], 2012). Ruland and Moore (1998) developed the middle-range Theory of the Peaceful End of Life from standards of care for terminally ill patients. In the work, the theorists observed that relational statements of the standards needed to be more specifically defined to make them applicable for empirical testing (McEwen \& Wills, 2011). Standards of care offer a promising approach for development of middle-range prescriptive theories because of their empirical base in clinical practice and their focus on linkages between interventions and outcomes (Ruland \& Moore, 1998). The authors of the Theory of the Peaceful End of Life connected nursing care to patient outcomes by using established standards of care in the theory. The standards of care of the peaceful end of life were established in a university hospital in Norway, where caring for terminally ill patients was part of the daily experience for nurses. The main focus for standard development was not on the final instance of dying itself, but on contributing to peaceful and meaningful living in the time that remained for the patients and their significant others (Ruland \& Moore). The authors examined the 16 outcome criteria of the standards of care and reduced them by common themes to establish five outcome indicators for their proposed theory (Figure 1). 


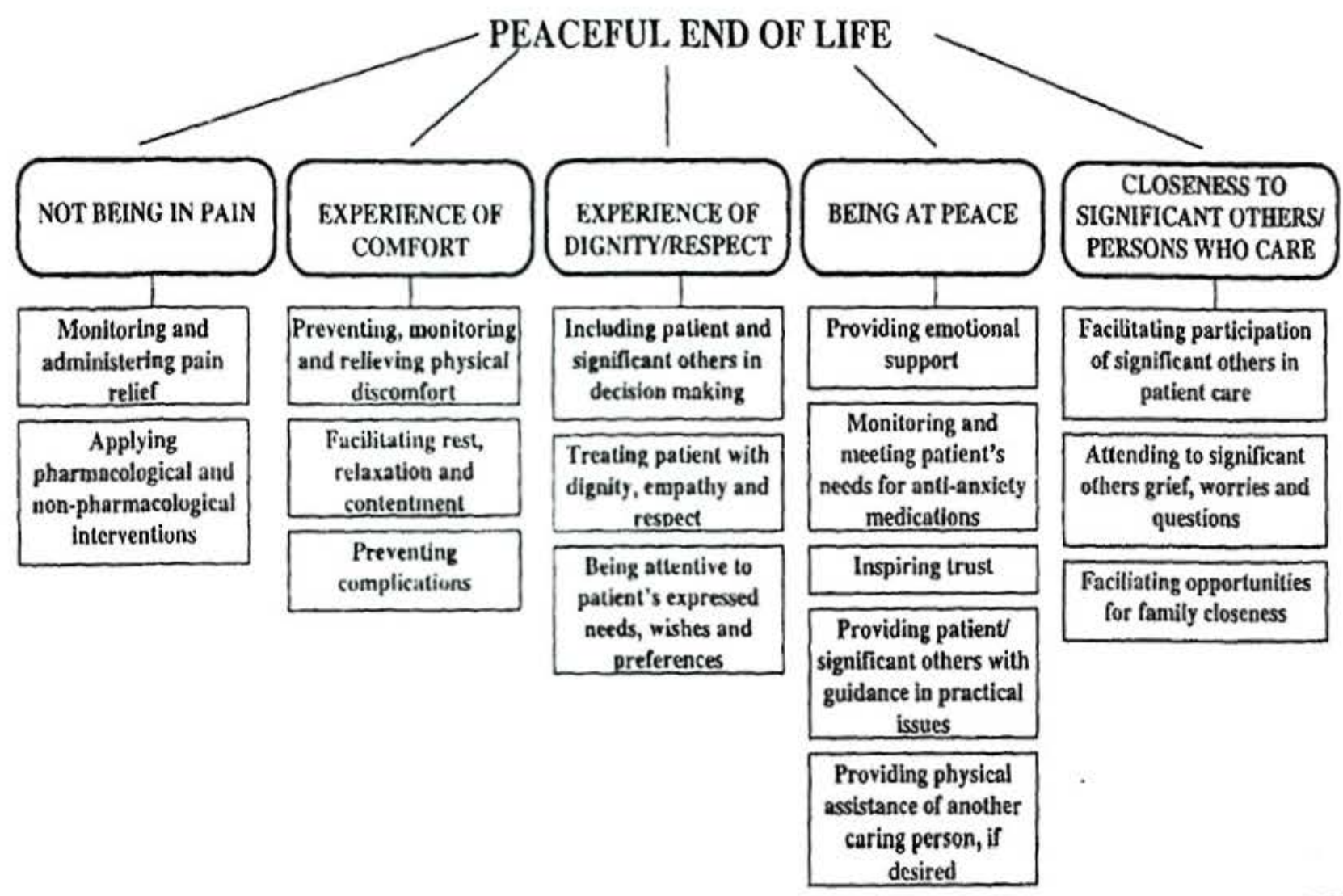

Figure 1. Relationship between the concepts of the theory (Ruland \& Moore, 1998).

Relational statements in a theory must be measurable, have the ability to produce testable hypotheses, and guide practice. The Theory of the Peaceful End of Life meets these requirements. All relationships between nursing interventions and outcomes indicators in the theory can be measured (Ruland \& Moore, 1998). Because of the measurability of the theory of peaceful end of life, it is a perfect fit for the program evaluation of palliative care provided on a MICU.

Next, the CDC framework will be reviewed.

\section{A Framework for Program Evaluation}

The Framework for Program Evaluation was developed by the CDC to evaluate the process of the program evaluation. The framework is a practical, non-prescriptive 
tool, designed to summarize and organize essential elements of program evaluation (CDC, 2012). Effective program evaluation is a systematic way to improve and account for program actions involving methods that are useful, feasible, ethical, and accurate. The CDC framework is illustrated in Figure 2.

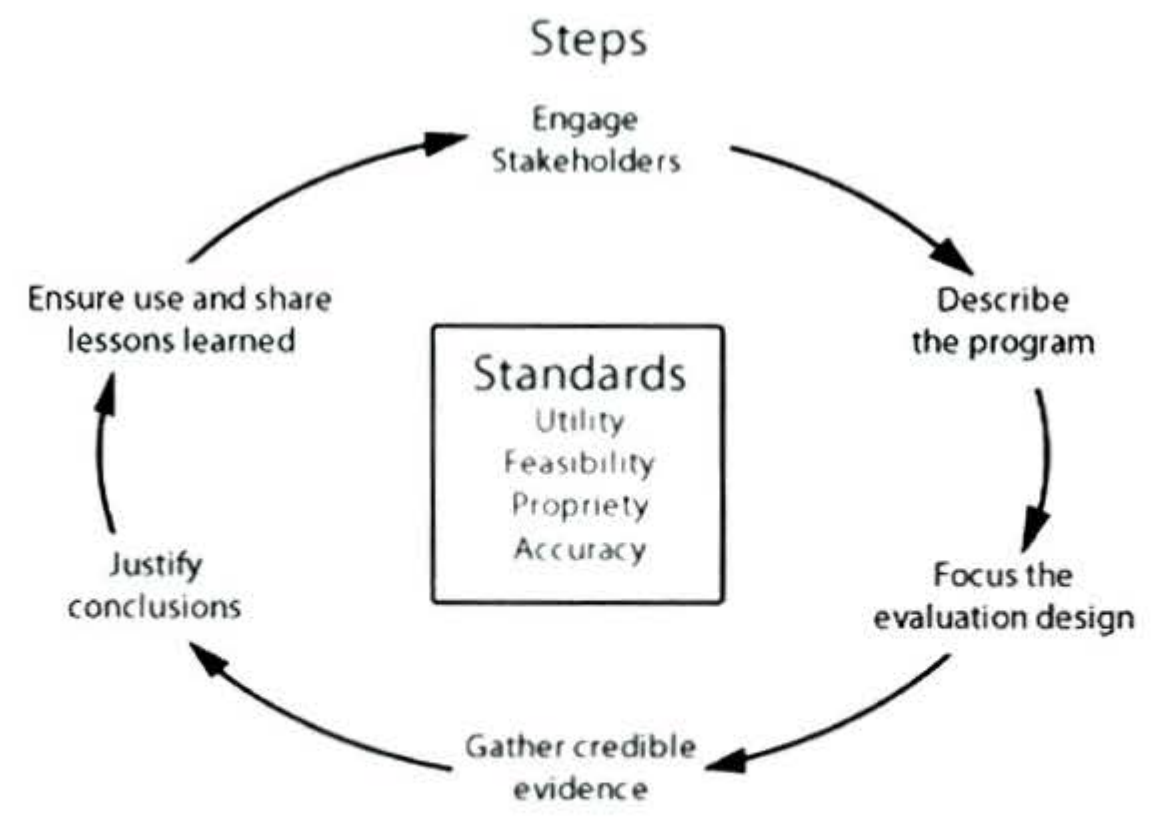

Figure 2. A Framework for Program Evaluation (CDC, 2012).

The CDC framework includes five steps: engage the stakeholders; describe the program; focus the evaluation design: gather credible evidence; justify conclusion; and ensure use and share lessons learned. Description of the steps will be described below.

Engaging the stakeholders. The evaluation cycle starts with engaging the stakeholders, the personnel who are involved in or affected by the program, the primary users of the evaluation. When stakeholders are not engaged, evaluation findings might be ignored, criticized, or resisted because they do not address the stakeholders' questions or values. 
Describe the program. The program description sets the frame of reference for decisions in the evaluation. Through the description, comparisons with standards of care can be established and attempts can be made to connect program elements to their effect.

Focus the evaluation design. The direction and process of the evaluation must be focused to assess issues of greatest concern to stakeholders while using time and resources as efficiently as possible. The components of the evaluation design include the purpose, identification of users, and uses of the project.

Gather credible evidence. The source of the information gathered and any evaluation tools used, need to be considered, to provide validity and reliability to the findings of the program evaluation.

Justify conclusions. Having the prior support from the major stakeholders and the utilization of valid and reliable evaluations tools will help justify the conclusions of the program evaluation.

Ensure use and share lessons learned. Assuming that lessons learned in the course of an evaluation will automatically translate into informed decision-making and appropriate action would be naive. Deliberate effort is needed to ensure that the evaluation processes and findings are used and disseminated appropriately. (CDC, 2012) Next, the methodology of the research will be presented. 


\section{Methodology}

\section{Purpose}

The purpose of this research project was to determine whether the patients on a MICU were offered appropriate palliative care. There were two goals of the project. The first was to identify those patients at high risk for unmet tertiary palliative care needs and in need of a Palliative Care Consultation using the Palliative Care Service Tool (see Appendix C). The second goal was to assess if those patients in need of basic primary palliative care services needs were met, as evaluated through the Care and Communication Bundle (see Appendix B).

\section{Research Question}

The research question was: Were the patients on a Medical Intensive Care Unit (MICU) appropriately referred to palliative care services?

\section{Sample/participants}

The sample consists of all adult patients $>18$ years of age admitted to MICU with a length of stay $\geq 5$ days. Five days was chosen to be in accordance with the guidelines of the Care and Communication Bundle to address the basic palliative needs of the patients. All patients that are less than 19 years old or whose length of stay was less than five days were excluded.

\section{Site}

This research took place at a 653 bed acute care facility, located in Providence, Rhode Island, and a major teaching hospital for Brown University Medical School. The data were collected from the records of patients admitted to the 18 bed medical intensive 
care unit, serving adult patients with acute and chronic medical diagnoses.

\section{Design}

The program evaluation was a retrospective chart review of 250 patients admitted to the Medical Intensive Care Unit (MICU) from January 1, 2013 to March 31, 2013. All patient records were contained within the electronic medical record (EMR). Charts were evaluated utilizing the Care and Communication Bundle of nine process measures of quality (see Appendix B) and the Palliative Care Service Tool (see Appendix C).

\section{Measurement}

The student researcher developed a data collection tool what was used to collect demographic information, the Patient Data Collection Sheet (Appendix D). The student researcher also collected data with the Care and Communication Bundle Data Collection Tool (Appendix E), which recorded the nine process measures of quality for primary palliative care that were established by the AHRQ for each patient. The timeframe for the nine quality measures includes: on day one, the identification of an appropriate medical decision maker, advanced directive status, addressing cardiopulmonary resuscitation preference, distribution of an information leaflet, pain assessment, and pain management; by day three, offering social work support and offering spiritual support; and by day five, conducting an interdisciplinary family meeting.

The Palliative Care Service Consult Tool (Appendix C) was also used, which is a scoring tool developed by the Palliative Care Consultation Team at the hospital. The tool contained five sections; the first section asked the question, "Would you be surprised if this patient were alive in one year $(\mathrm{yes}=3, \mathrm{no}=0$ ). The second section identified basic 
disease processes: cancer; advanced COPD; neurological diseases; end-stage-renal disease; advanced CHF; if the patient was not a candidate for curative surgery; and greater than three hospitalizations, emergence department visits or ICU stays in the past year. Each disease or condition received two points. The third section identified any uncontrolled symptoms or clinical conditions: pain; dyspnea; nausea; bowel obstruction; weight loss; constipation; prolonged ventilator support; and a history of cardiac arrest. Each symptom received two points. The fourth section assessed the anticipated functional status of the patient at the time of discharge using the Eastern Cooperative Oncology Status (ECOG). Grading is scored from 0-4, with 0-1 returning to pre-disease activities without restrictions, and 4 being completely disable. The fifth section addressed any psychological issues of the patient or the family, such as the need to discuss end-of-life issues, the need to evaluate the need for possible hospice referral, artificial nutrition or hydration requested or considered, or unrealistic goals or expectations, each receiving two points. The total points were added up for each patient to evaluate the need for a palliative care consult; $9-11$ points would suggest to consider a consult and greater than 12 would suggest to strongly consider a palliative care consult.

\section{Procedures}

The procedures will be outlined using the CDC framework.

Engage the stakeholder. The evaluation cycle starts with engaging the stakeholders, which for this project was the Medical Director of the Medical Intensive Care Unit (MICU), the Clinical Nurse Manager, the Chief Nursing Officer, the medical staff on the MICU, and the nursing staff, the personnel who were involved in or affected 
by the program, and the primary users of the evaluation. The research proposal had previously been submitted and been approved by the Institutional Review Board, both at Lifespan and Rhode Island College.

Describe the program. The program description set the frame of reference for the decisions in the evaluation. Through the description, comparisons with the standards of care were established and attempts were made to connect program elements to their effect. The program that was used on the MICU was a "consultative model." There was a trigger criteria, the Palliative Care Service Tool (Appendix C), that was used to identify those patients in need of tertiary palliative care, and a palliative care consult. The second part was to measure if all patients had their primary palliative care needs met using the Care and Communication Bundle (Appendix B).

Focus the evaluation design. The direction and purpose of the evaluation was to focus on assessing the issues of greatest concern to the stakeholders while using time and resources as efficiently as possible. There were two questions that were asked; whether the patients that were identified by the Palliative Care Service Tool (Appendix C) as in need of a palliative care consult received a consult, and second, if all patients had their primary palliative care needs met as established by the Care and Communication Bundle (Appendix B). The patients' EMR was accessed to collect the required information, including the patients' demographic data contained in the Patient Data Collection Sheet (see Appendix D), and to assess and score if the patients' primary and tertiary palliative care needs were met using the Care and Communication Bundle Data Collection (Appendix E) and the Palliative Care Service Consult Tool (Appendix C). 
Gather credible evidence. The student researcher completed all data collection, which was performed between July 25, 2014 and September 5, 2014. The student researcher was able to access an electronic list of all patients admitted to the MICU during the time of January 1, 2013 and March 31,2013. Only those patients meeting the inclusion criteria had their records accessed through the electronic medical record (EMR) system located on the MICU for further data collection. Two hundred fifty EMRs were reviewed to obtain 50 subjects. The demographic information from the EMR was collected and recorded on the Patient Data Collection Sheet (Appendix D), including the patients' age, gender, length of stay, admitting diagnosis, and past medical history. For patient confidentiality, the patients' information that was collected was kept on an encrypted thumb drive and secured in a locked locker. In addition to the demographic information, data contained in the Care and Communication Bundle (Appendix B) of nine process measures of quality was recorded on the Care and Communication Bundle Data Collection Sheet (Appendix E), and the palliative score was recorded on the Palliative Care Service Tool (Appendix (). Using established evaluation tools, the Care and Communication Bundle Appendix B) and the Palliative Care Service Tool (see Appendix C), provided validity and reliability to the findings of the program evaluation. These tools were available to the staff on MICU for the evaluation of patient palliative care needs.

Justify conclusions. Using the evaluation tools, the author was able to establish the percentage of patients who received a palliative care consult for their tertiary 
palliative care needs, the percentage of patients who had all their primary palliative care needs met, and identify those process measures of quality that were not met.

Ensure use and share lessons learned. Deliberate effort was needed to ensure that the evaluation process and findings are used and disseminated appropriately. The findings of the evaluation was shared with the major stakeholders, the medical director, the clinical manager, and the medical and nursing staff through an educational in-service that included a review of the tools that were used in the evaluation and the findings. 


\section{Results}

The total number of charts reviewed was 250 , of which 50 met the inclusion

criteria. There were 27 males (54\%) and 23 females (46\%); the ages ranged from 21-92

years old. Major diagnostic categories identified from the 50 charts are presented in Table

1.

Table 1 Major Diagnostic Categories

\begin{tabular}{|l|l|}
\hline Major Diagnostic Categories & Patients (N=50) \\
\hline Respiratory Failure & $18(36 \%)$ \\
\hline Sepsis/Shock & $13(26 \%)$ \\
\hline Stroke/neurological changes & $8(16 \%)$ \\
\hline PEA/cardiac arrest & $4(8 \%)$ \\
\hline GI Bleed & $2(4 \%)$ \\
\hline $\begin{array}{l}\text { Other (DKA, lactic acidosis, drug } \\
\text { overdose }\end{array}$ & $5(10 \%)$ \\
\hline
\end{tabular}

The nine quality process measures of the Care and Communication Bundle and the percent compliance are presented in Table 2 .

Table 2 Care and Communication Bundle Data Collection

\begin{tabular}{|ll|l|}
\hline Bundle Categories & Patients $(\mathbf{n}=\mathbf{5 0})$ \\
\hline 1) & Medical decision maker & $49(98 \%)$ \\
\hline 2) Advanced directive status & $50(100 \%)$ \\
\hline 3) CPR preference & $50(100 \%)$ \\
\hline 4) Information leaflet & $49(98 \%)$ \\
\hline 5) Pain assessment every 4 hours & $40(80 \%)$ \\
\hline 6) Pain management & $38(76 \%)$ \\
\hline 7) Social work support offered & $20(40 \%)$ \\
\hline 8$)$ Spiritual support offered & $37(74 \%)$ \\
\hline 9) Interdisciplinary family meeting & $46(92 \%)$ \\
\hline
\end{tabular}


Compliance with five of the components was greater than $90 \%$, with the remaining four less than $90 \%$. The four quality process measures that were less than $90 \%$ were pain assessment $(80 \%)$, pain management $(76 \%)$, social work support offered (40\%), and spiritual support offered (74\%).

The Palliative Care Team has a tool in place, the Palliative Care Service Consult Tool, to identify patients in need of tertiary palliative care. Table 3 illustrates the total palliative care score of the 50 charts reviewed.

Table 3

Total Palliative Care Score

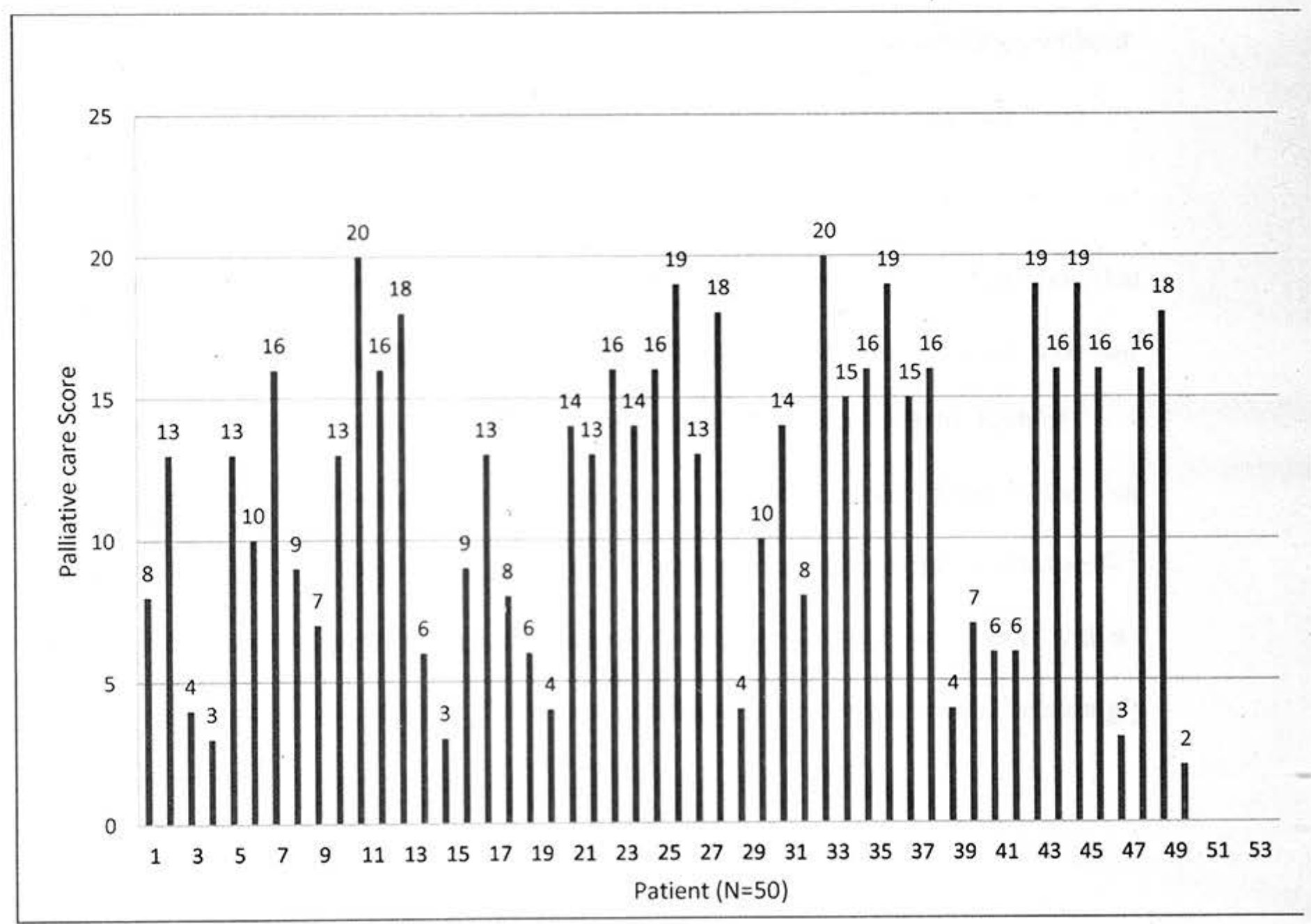


The following scale was used to interpret the total score:

-Total Score $\leq 8$ problem directed: consult if desired

-Total Score 9-11: consider consult

Total Score $\geq 12$ : strongly consider consult

Of the 50 records reviewed, there were $17(34 \%)$ with palliative care scores

less than or equal to eight, indicating consult if desired. Five of the 17 had a history of advanced disease, including two with (COPD), two with end-stage renal disease (ESRD), and one with a diagnosis of stroke. Sixteen of the subjects (94\%) had uncontrolled symptoms or clinical conditions, including dyspnea (4), pain (5), and prolonged ventilator support (7). The number of subjects anticipated to return to pre-disease activities without restriction was six $(35 \%) ; 10(58 \%)$ were identified as capable of most self-care activities, and one (6\%) was capable of only limited self-care activities.

Four records $(8 \%)$ revealed palliative care scores between 9 and 11, indicating that a consult should be considered. Of the four, three subjects had advanced disease: one had ESRD, requiring hemodialysis, and two had congestive heart failure (CHF), both of whom had uncontrolled dyspnea requiring prolonged ventilator support. Three of the four subjects were anticipated to return to full activity, with one capable of only limited selfcare activities. Table 4 on the next page illustrates the composition of the patients with a palliative care score greater than 12 , for which a palliative care consult should be strongly considered. 
Table 4

Patients with Palliative Care Scores $>12$

Palliative Care Composition

Patients $(\mathbf{N}=29)$

Advanced Disease

1) Cancer (metastatic/recurrent)

$6(21 \%)$

2) Advanced COPD

$8(28 \%)$

3) Neurological disease

$9(31 \%)$

4) End-stage renal disease

$5(17 \%)$

5) Other

$1(3 \%)$

6) Patients with $\geq 2$ advanced diseases

$20(69 \%)$

Uncontrolled symptoms
1) Pain
$6(21 \%)$
$15(51 \%)$
2) Dyspnea
$8(28 \%)$
3) Prolonged ventilator support
$16(55 \%)$
4) Patients with $\geq 2$ uncontrolled symptoms

Anticipated functional status

1) Return to full activity

$0(0 \%)$

$2(7 \%)$

2) Capable of most self-care

$21(72 \%)$

3) Capable of only limited self-care

4) Completely disable

$6(21 \%)$

Psychological issues (end-of-life, hospice, unrealistic goals)

1) End-of-life issues

$16(55 \%)$

2) Hospice referral

$9(31 \%)$

3) Request for artificial nutrition

$4(14 \%)$

There were 29 subjects $(58 \%)$ whose palliative care scores were greater than 12 and who should have been strongly considered for a palliative care consult. Of those, only two $(6 \%)$ had a palliative care consult ordered. All of the subjects in this group were diagnosed with advanced disease; 20 (69\%) were diagnosed with two or more advanced comorbidities, and 16 subjects (55\%) had two or more uncontrolled symptoms.

The overwhelming majority of the subjects $(93 \%)$ had an anticipated significant decline in functional status and/or "poor quality of life" as described by the patient and family, 
and were capable of only limited self-care or completely disable. Another important aspect of this group of subjects was that $25(86 \%)$ had end-of-life concerns/goals of care or inquires about a hospice referral.

Next, the summary and conclusions will be presented. 


\section{Summary and Conclusions}

More people die in the intensive care unit (ICU) setting than in any other part of the hospital. For many patients and families, the ICU experience is one that is associated with a significant burden of symptoms and may result in long term functional decline and an unacceptable quality of life. Critical illness was once clearly distinguished from 'terminal illness'. Patients were brought to ICUs to save their lives, not to provide end-oflife care. For most patients, the discussion about comfort and end-of-life goals moved to the center of attention only after hope of cure or substantial prolongation of life was lost (Nelson \& Danis, 2001). Palliative care was postponed until death was known to be near.

In the summer of 2001, the Institute of Medicine (IOM), in partnership with the National Research Council, recognized the need for improvement of end-of-life and palliative care in the report Improving Palliative Care in Cancer (Foley \& Gelband, 2001). Despite major advances in the management of some cancers, $50 \%$ of all patients diagnosed with cancer were dying within a few years. Dying from cancer had become synonymous with a spectrum of symptoms including pain, labored breathing, distress, nausea, confusion, and other physical and psychological conditions that go untreated or undertreated and vastly diminish the quality of those lives (Chertkov et al., 2001). Palliative care focuses on complex pain and symptom management, communication about care goals, alignment of treatments with patient values and preferences, transitional planning, and support for the family. This type of care is seen as an essential component of comprehensive care for patients with critical illness, including those receiving aggressive intensive care (Nelson et al., 2010). 
Nelson et al. (2010) conducted a literature review of MEDLINE database from inception to April 2010 using the terms 'intensive care' and 'palliative care'. The authors reviewed the existing data with a focus on models that have been used to structure clinical initiatives to enhance palliative care for critically patients in the ICU and their families. The authors commented that although prior literature illustrated why palliative care could be improved, guidance on how this might be effectively accomplished in practice remained limited. Nelson et al.'s analysis of the literature focused on practical approaches to ICU palliative care and introduced two main models for ICU palliative care integration: (1) the "consultative model," which utilizes the medical specialty of a palliative care team in the care of the critically ill intensive care patients and their families; and (2) the "integrative model," which seeks to root the principles of palliative care into daily practice of the intensive care team for all patients and families facing critical illness.

The current practice on the MICU which served as the study site utilizes a combination of the consultative and integrative models. Patients with tertiary palliative care needs are identified through a palliative care criteria tool developed by the Palliative Care Team; recommendations as to receiving a palliative care consult are identified. The Palliative Care Service Consult Tool (Appendix B) identifies the patient's co-morbidities, any uncontrolled symptoms or clinical conditions, the patient's anticipated functional status upon discharge, any psychological issues of the patient or the family, and asks the question "Would you be surprised if this patient were alive in one year?" A detailed 
description of the tool along with the scoring system can be found in the methods section under measurement.

Primary palliative care needs are addressed by the MICU staff through the use of the Care and Communication Bundle (Appendix B). The Care and Communication Bundle measures the patients' primary palliative care needs using nine process measures of quality: medical decision maker identified; advanced directive status established; CPR preference; information leaflet given; pain assessment and pain management; social work support offered; spiritual support offered; and interdisciplinary family meeting.

The purpose of this project was to determine if patients on MICU were receiving appropriate palliative care and evaluating the program in place using the established tools mentioned above. This program evaluation was guided by the Theory of Peaceful End of Life (Rulund \& Moore, 1998) and the Framework for Program Evaluation (CDC, 2012). The program evaluation was a retrospective chart review of patients admitted to the Medical Intensive Care Unit (MICU) from January 1, 2013 to March 31, 2013. The total number of charts reviewed was 250 , of which 50 met the inclusion criteria: subjects $>18$ years of age admitted to MICU with a length of stay $\geq 5$ days. Five days was chosen to be in accordance with the guidelines of the Care and Communication Bundle to address the basic palliative needs of the patients. All patients that were less than 19 years old or whose length of stay was less than five days were excluded.

There were 27 males (54\%) and 23 females (46\%) in the sample; ages ranged from 21-92 years old. The major diagnostic categories identified from the 50 charts were respiratory failure $(36 \%)$, sepsis/shock $(26 \%)$, stroke/neurological changes $(16 \%)$, 
PEA/cardiac arrest (8\%), GI bleed (4\%), and other diagnoses (10\%). All patient charts were reviewed for compliance with the nine quality process measures of the Care and Communication Bundle that were described above. Compliance with five of the components was greater than $90 \%$, with the remaining four less than $90 \%$. The four quality process measures that were less than $90 \%$ were pain assessment $(80 \%)$, pain management $(76 \%)$, social work support offered (40\%), and spiritual support offered (74\%). These findings suggest what the literature has demonstrated: the areas that providers need to improve on are pain and symptom management and offering spiritual and social work support.

Grant et al. (2009) commented on the current status of palliative care and how it was improving the care for patients with serious and life-threatening cancer through the creation of national guidelines for quality care, multidisciplinary educational offerings, research endeavors, and resources made available to clinicians. The authors commented that when serious and life-threatening illness occurred, the patients voiced their priorities as pain and symptom control, avoidance of prolongation of the dying process, a sense of control, and an opportunity to strengthen relationships with loved ones. The authors reported that moderate to severe pain was documented in $60 \%$ of patients with colon cancer and $57 \%$ of patients with lung cancer. On average, it took between eight and 14 days to get pain under control. In that same study by Grant et al., family members also reported poor emotional support and a lack of respectful treatment.

The second part of this study aimed to identify patients in need of tertiary palliative care by using a scoring tool, the Palliative Care Service Consult Tool 
(Appendix C), which was described above. Of the 50 records reviewed, there were 17 (34\%) with palliative care scores less than or equal to eight, indicating a palliative consult, if desired. The number of subjects anticipated to return to pre-disease activities without restriction was six (35\%); ten ( $58 \%)$ were identified as capable of most self-care activities, and one (6\%) was capable of only limited self-care activities. Four records (8\%) revealed palliative care scores between 9 and 11, indicating that a palliative consult should be considered. This group of subjects had advanced disease, including CHF and $\mathrm{CKD}$, with three of the four subjects anticipated to return to full activity.

There were 29 subjects ( $58 \%$ ) whose palliative care scores were greater than 12 and who should have been strongly considered for a palliative care consult. Of those, only two $(6 \%)$ had a palliative care consult ordered. All of the subjects in this group were diagnosed with advanced disease; $20(69 \%)$ were diagnosed with two or more advanced comorbidities and 16 subjects $(55 \%)$ had two or more uncontrolled symptoms. The overwhelming majority of the subjects (93\%) had a significant anticipated decline in functional status and/or "poor quality of life" as described by the patient and family, and were capable of only limited self-care or completely disable. Another important aspect of this group of subjects was that $25(86 \%)$ had end-of-life concerns/goals of care or inquires about a hospice referral.

The results of this study are limited by the relatively small sample size of 50 subjects and the fact that this was just one intensive care unit in one hospital. The Palliative Care Service Tool incorporates some provider subjectivity in assessing the patients, as illustrated by the first question on the tool, "Would you be surprised if this 
patient were alive in one year?" and by asking the provider to predict their patients' anticipated functional status upon discharge. However, the tool adopted by the hospital is the same tool developed by the Center to Advance Palliative Care (CAPC), which is the nation's leading resource for palliative care development and growth, and is used nationally.

These findings suggest that providers may benefit from not only a re-evaluation of their knowledge of palliative care and the tools available to guide them, but also their personal view and attitude concerning palliative care. This may enable providers to better serve the needs of their critically ill patients who may be at the end of their lives. Palliative care focuses on providing patients with relief from symptoms, pain and stress of a serious illness, and applies to any medical diagnosis. The goal is to improve quality of life for both patient and family (CAPC, 2006, Defining Palliative Care, para. 1). High quality care for intensive care patients and their families includes palliative care. As demonstrated by the results, many patients were identified by the palliative care tool as candidates for a consult and these patients and families likely could have benefitted from one but did not receive it. It is possible that the language connected to the scoring system is not stated strongly enough. For example, instead of stating that for those patients with scores greater than 12 the provider should 'strongly consider a consult,' perhaps using the language 'should receive a palliative care consult' would be useful in generating more consults.

Next, recommendations and implications will be presented and discussed. 


\section{Recommendations and Implications}

For patients on an intensive care unit, it is paramount that palliative care needs are assessed starting on day one. This research evaluated both primary and tertiary palliative care of patients in a medical intensive care unit. Consistent with the current literature, "patients with a serious illness and their families receive poor quality medical care, characterized by untreated symptoms, unmet psychosocial and personal care needs, a great burden for caregivers, and low patient and family satisfaction" (Meier, 2011, p.351).

The Care and Communication Bundle was in place at the study institution to address the patients' primary palliative care needs. The palliative care team had also adopted a tertiary palliative care tool that mirrors a tool developed by the CAPC. The Care and Communication Bundle was incorporated into the patients' EMR. However, the tertiary tool was not included. The literature clearly supports that a tool to address tertiary needs be adopted (CAPC, 2007). Given the current emphasis on quality and the importance of patient satisfaction, palliative care as the standard should be incorporated into hospital policies.

Clinicians tend to perceive palliative care as the alternative to life-prolonging or curative care or what we do when there is nothing more that we can do, rather than a simultaneously delivered adjunct to disease-focused treatment (Kelly \& Meier, 2010). A question that arises is 'Does the culture of healthcare need to be changed, or does it need 
to be educated?' Evidence supports the benefits of palliative care. For example, in one study, cancer patients who understood their terminal prognosis and were provided palliative care along with standard care demonstrated improved mental health and quality of death as well as caregivers with higher bereavement adjustment (Meier, 2011).

Meeting the palliative care needs of our patients is a key quality initiative. The Center to Advance Palliative Care (CAPC), in identifying tertiary palliative care needs for patients, has recognized four areas of concern when evaluating patients: the patient's co-morbidities; uncontrolled symptoms or clinical conditions; anticipated functional status; and psychological issues of the patient or family concerning end-of-life concerns (2012). These areas should be explored during the admission interview. During this process, no matter how it is approached, providers need to be ethically and culturally sensitive to patients and their families.

Assuming that lessons learned in the course of an evaluation will automatically translate into informed decision-making and appropriate action would be naïve.

Deliberate effort is needed to ensure that the evaluation process and findings are used and disseminated appropriately (CDC, 2012). The evaluation cycle starts and ends with engaging the stakeholders, the personnel who are involved in or affected by the program, the primary users of the evaluation. The primary stakeholders in this program evaluation were the Medical Director of the Medical Intensive Care Unit (MICU), the Clinical Nurse Manager, the Chief Nursing Officer, the medical staff on the MICU, and the nursing staff. The tertiary palliative care tool for evaluation is in place, but the results show that it is not being utilized. Results of this program evaluation will be reported back to the 
stakeholders, namely the Medical Director, the Chief Nursing Officer, and the Clinical Manager. Recommendations for practice change include making the tool part of the patients' electronic medical record and admission assessment. The second stage of the process would include staff education for the medical and nursing disciplines on the process and use of the measures in identifying patients in need of tertiary palliative care and a possible palliative care consult. The final stage would be a re-evaluation of the use of the tool six months after staff education has been completed.

Advanced practice nurses can be key in educating, implementing and evaluating a comprehensive program to ensure that palliative care is the standard of care for all patients admitted to a hospital and/or an ICU. Advanced practice nurses have key roles in the care of patients who are nearing death and/or those living with a disabling chronic disease. It starts with the education of the nursing staff about the tools in place to aid in identifying those patients with palliative care needs and assisting and role modeling communication skills with patients at the end of their life. The palliative care assessment measures are available and are evidence-based, and they need to be shared and disseminated to the staff. Role modeling may be particularly important, as a culture change for the staff will likely be indicated, because as providers, we have been trained to provide the most technically advanced care available to 'save' our patients. Fins (2006) suggested that instead of letting available technology drive the goals of care, we should let the goals of care drive the therapy.

Palliative care should be considered standard of care for all patients admitted to the intensive care area. The APRN is at the center of this movement to educate, to 
influence the present culture of the unit, and to incorporate a collaborative, interdisciplinary approach to ensure that all patients' palliative care needs are met. Compliance with the evidence-based interventions should no longer be optional, but be incorporated into the standards of care. This might have to start with a policy change to get the necessary assessment tools incorporated into the patients' EMR in order to better ensure that the patients' needs are identified.

Due to the diverse demographic make-up in our patient population, providers are increasingly caring for patients from cultural backgrounds other than their own. Examples include cultures with different beliefs, values, and health care practices which become particularly relevant at the end of life. Proper end-of-life care may be compromised by disagreements between providers and patients or by decisions that the provider does not understand. This could be an area of future research to further improve end-of-life care for the culturally diverse patient population. Providers need to be sensitive to cultural differences and develop the skills necessary to work with patients from diverse backgrounds. Community and cultural ties provide a source of great comfort as patients and families prepare for death. Providers should assess the cultural background of each patient and inquire about values that may affect care at the end-oflife.

A tertiary palliative care screening tool should be incorporated into the admission process for all patients admitted to an ICU, but this is only one small aspect of the issue at the institutional level. Palliative care needs to be integrated into national policy. Well written policies lay the groundwork for an effective health care system and society 
(Stjernsward, Foley, and Ferris 2007). National and state policies on palliative care would facilitate the implementation of palliative care programs aimed at providing care for all people in need of these services, and ensure equitable access to end-of-life care. The lack of these policies can lead to unnecessary suffering and costs for patients, families, and the healthcare system. It has been estimated that three-quarters of cancer patients worldwide are incurable when diagnosed. Stjernsward et al. (2007) commented that because the size of the problem and the suffering associated with cancer is enormous, the development of a national cancer control policy is an effective point of entry to begin integrating palliative care into a national health care system. Advanced practice nurses have a key role in advocating for policy changes not only in their institutions that help their patients meet their goals of care, especially at the end of their lives, but more importantly at the national and state level, affecting change that would impact all patients at the end of their lives. 


\section{References}

Agency for Healthcare Research and Quality (2006). Voluntary hospital association care and communication bundle: Care and communication quality measures. Retrieved from: www.qualitymeasures.ahrq.gov/summary/summary.aspx?doc_id=9943

Amitabha Hospice Service (2009). A brief history of hospice care. Retrieved from:www.amitabhahospice.org/public/who-we-are/general_history.php

Angus, D.C., Barnato, A.E., Lindle-Zwirble, W.T., Weissfeld, L.A., Watson, R.S., Rickert, T. \& Rubenfeld, G.D. (2004). Use of intensive care at the end of life in the united states: An epidemiologic study. Critical Care Medicine, 32(3), 638642.

Azoulay, E., Pochard, F., Chevret, S., Jourdain, M., Bornstain, C., Wernet, A., \& Lemaire, F. (2002). Impact of a family information leaflet on effectiveness of information provided to family members of intensive care unit patients: A multicenter, prospective, randomized controlled trial. American Journal of Respiratory Critical Care Medicine, 165(4), 438-442.

Campbell, M.L. \& Guzman, J.A. (2003). Impact of a proactive approach to improve endof-life care in a medical icu. Chest, 123, 266-271.

Center to Advance Palliative Care (2006). Defining palliative care. Retrieved from: www.capc.org/building-a-hospital-based-palliative-care-program/case/definingpc 
Center to Advance Palliative Care (2007). Fact sheet on the "care and communication bundle" of icu palliative care quality measures. Retrieved from: www.capc.org/palliative-care-across-the ...care-unit/icu-bundle.doc

Center to Advance Palliative Care (2011). About capc. Retrieved from: www.capc.org/about-capc

Center to Advance Palliative Care (2012). Growth of palliative care in u.s. hospitals 2012 Snapshot. Retrieved from: www.reportcard.capc.org/capc-growth-analysissnapshot-2011.pdf

Centers for Disease Control and Prevention (2012). Framework for program evaluation. Retrieved from: www.cdc.gov/eval/framework/index.htm

Chertkov, L., Cleeland, C.S., Freyer, D.R., Friebert, S., Hilden, J.M., Himelstein, B.P.,...Teno, J.M., (2001). Improving palliative care for cancer. Washington, DC: National Academy Press.

Connors, A.F., Dawson, N.V., Desbiens, N.A., Fulkerson, W.J., Goldman, L., Knaus, W.A.,...Oye, R.K. (1995). A controlled trial to improve care for seriously ill hospitalized patients. The Journal of the American Medical Association, 274(20), 1591-1598.

Dumitrescu, L. (2006). Palliative care in romania. Romana: Romanian Revista Medicala Romana. 
Field, M.J. \& Cassel, C.K. (Eds) (1997). Approaching death: Improving care at the end of life. Washington, DC: National Academy Press.

Field, M.J. \& Cassell, C.K. (Eds) (1998). Approaching death: Improving care at the end of life. New England Journal of Medicine, 339, 274-275.

Fins, J.J. (2006). A palliative ethic of care: clinical wisdom at life's end. Mississauga, Ontario: Jones \& Bartlett Publishing.

Foley, K.K. \&Gelband, H. (Eds) (2001). Improving palliative care for cancer. Washington, DC: National Academies Press.

Grant, M., Elk, R., Ferrell, B., Morrison, R.S. \& Von Gunten, C.F. (2009). Current status of palliative care, education, and research. CA: A Cancer Journal for Clinicians, 59(5), 327-335.

Hospice Education Institute (2002). A short history...from middle ages to the $21^{\text {st }}$ century. Retrieved from: www.hospiceworld.org/history.htm

Kelly, A.\& Meier, D. (2010). Palliative care - a shifting paradigm. New England Journal of Medicine, 363, 781-782.

Levy, M.M., (2001). Compassionate end-of-life care in the intensive care unit. Critical Care Medicine, 29(2), 1-2.

Lunney, J.R., Foley, K.M., Smith, T.J., \& Gelband, H. (2003). Describing death in america, what we need to know. Washington, DC: National Academy Press. 
McEwen, M. \& Wills, E.M. (2011). Theoretical basis for nursing. Philadelphia, PA: Lippincott Williams \& Wilkins.

Meier, D., (2011). Increased access to palliative care and hospice services: opportunities to improve value in health care. Milbank Quarterly, 89(3), 343-380.

Morgan, D. (2009). Caring for dying children: Assessing the needs of the pediatric palliative care nurse. Pediatric Nursing, 35(2), 86-91.

National Consensus Project (2013). Clinical practice guidelines for quality palliative care.Retrieved from; www.nationalconsensusproject.org/guideline.pdf

Nelson, J.E. (2006). Identifying and overcoming the barriers to high-quality palliative care in the intensive care unit. Critical Care Medicine, 34(11), S324-330.

Nelson, J.E., Bassett, R., Boss, R.D., Brasel, K.J., Campbell, M.L., Cortez, T.B.,...Weissman, D.E. (2010). Models for structuring a clinical initiative to enhance palliative care in the intensive care unit: A report from the ipal-icu project (improving palliative care in the icu). Critical Care Medicine, 38(9), 1765-1772.

Nelson, J.E., Cortez, T.B., Curtis, J.R., Lustbader, D.R., Mosenthal, A.C., Mulkerin, C.,...Puntillo, K.A. (2011). Integrating palliative care in the icu, The nurse in a leading role. Journal of Hospice \& Palliative Nursing, 13(2), 89-94.

Nelson, J.E. \& Danis, M. (2001). End-of-life care in the intensive care unit: Where are we now? Critical Care Medicine, 29(2), 2-9. 
Nelson, J.E., Mulkerin, C.M., Adams, L.L., \& Pronovost, P.J. (2005). Improving comfort and communication in the icu: a practical new tool for palliative care performance measurement and feedback. Quality Safe Health Care, 15, 264-271.

Nelson, J.E., Puntillo, K.A., Pronovost, P.J., Walker, A.S., McAdam, J.L., Ilaoa, D. \& Penrod, J. (2010). In their own words: Patients and families define high-quality palliative care in the intensive care unit. Critical Care Medicine, 38(3), 808-818.

North Simcoe Muskoka Palliative Care Network (2012). The origin of hospice palliative care. Retrieved from: www.palliativecarenetworknsm.ca/index.php/origin-ofhospice-Palliative-care

Penrod, J.D., Pronovost, P.J., Livote, E.E., Puntillo, K.A., Walker, A.S., Wallenstein, S.,...Nelson, J.E. (2012). Meeting standards of high-quality intensive care unit palliative care: Clinical performance and predictors. Critical Care Medicine, 40(4), 1105-1112.

Ruland, C., \& Moore, S. (1998). Theory construction based on standards of care: A proposed theory of the peaceful end of life. Nursing Outlook, 46, 169-175.

Stjernsward, J., Foley, K.M., \& Ferris, F.D. (2007). Integrating palliative care into national policies. Journal of Pain and Symptom Management, 33(5), 514-520.

Temel, J.S., Greer, J.A., Muzikansky, A., Gallagher, E.R., Admane, S., Jackson, V.A.,,...Lynch, T.J. (2010). Early palliative care for patients with metastatic nonsmall-cell lung cancer. The New England Journal of Medicine, 363(8), 733-742. 
Unroe, M., Kahn, J.M., Carson, S.S., Govet, J.A., Martinu, T., Sathy, S.J.,...Cox, C.E. (2010). One-year trajectories of care and resource utilization for recipients of prolonged mechanical ventilation. Annals of Internal Medicine, 153(3), 167-175.

Weissman, D. \& Meier, D. (2011). Identifying patients in need of a palliative care assessment in the hospital setting. Journal of Palliative Medicine, 14, 1-7.

World Health Organization (2004). Definition of palliative care. Retrieved from: www.who.int/Cancer/palliative/definition/en/ 


\section{Appendix A}

\section{Clinical Practice Guidelines for Quality Palliative Care \\ (National Consensus Project, 2013)}

\section{Domain 1: Structure and Processes of Care.}

Guideline 1.1 A comprehensive and timely interdisciplinary assessment of the patient and family forms the basis of the plan of care.

Guideline 1.2 The care plan is based on the identified and expressed preferences, values, goals, and needs of the patient and family and is developed with professional guidance and support for patient-family decision making. Family is defined by the patient.

Guideline 1.3 An interdisciplinary team (IDT) provides services to the patient and family consistent with the care plan. In addition to chaplains, nurses, physicians, and social workers, other therapeutic disciplines who provide palliative care services to patients and families may include: child-life specialists, nursing assistants, nutritionists, occupational therapist, recreational therapists, respiratory therapists, pharmacists, physical therapists, massage, art, and music therapists, psychologists, and speech and language pathologists.

Guideline 1.4 The palliative care program is encouraged to use appropriately trained and supervised volunteers to the extent feasible.

Guideline 1.5 Support for education, training, and professional development is available to the interdisciplinary team. 
Guideline 1.6 In its commitment to quality assessment and performance improvement, the palliative care program develops, implements, and maintains an ongoing data driven process that reflects the complexity of the organization and focuses on palliative care outcomes.

Guideline 1.7 The palliative care program recognizes the emotional impact of the provision of palliative care on the team providing care to patients with serious or lifethreatening illnesses and their families.

Guideline 1.8 Community resources ensure continuity of the highest quality palliative care across the care continuum.

Guideline 1.9 The physical environment in which care is provided meets the preferences, needs, and circumstances of the patient and family, to the extent possible.

\section{Domain 2: Physical Aspects of Care.}

Guideline 2.1 The interdisciplinary team assures and manages pain and/or other physical symptoms and their subsequent effects based upon the best available evidence.

Guideline 2.2 The assessment and management of symptoms and side effects are contextualized to the disease status.

\section{Domain 3: Psychological and Psychiatric Aspects of Care.}

Guideline 3.1 The interdisciplinary team assesses and addresses psychological and psychiatric aspects of care based upon the best available evidence to maximize patient and family coping and quality of life.

Guideline 3.2 A core component of the palliative care program is a grief and bereavement program available to patients and families, based on assessment of need. 


\section{Domain 4: Social Aspects of Care.}

Guideline 4.1 The interdisciplinary team assesses and addresses the social aspects of care to meet patient-family needs, promote patient-family goals, and maximize patientfamily strengths and well-being.

Guideline 4.2 A comprehensive, person-centered interdisciplinary assessment (as described in Domain 1, Guideline 1.1) identifies the social strengths, needs, and goals of each patient and family.

\section{Domain 5: Spiritual, Religious, and Existential Aspects of Care.}

Guideline 5.1 The interdisciplinary team assesses and addresses spiritual, religious, and existential dimensions of care.

Guideline 5.2 A spiritual assessment process, including a spiritual screening, history questions, and a full spiritual assessment as indicated, is performed. The assessment identifies religious or spiritual/existential background, preferences, and related beliefs, rituals, and practices of the patient and family; as well as symptoms, such as spiritual distress and/or pain, guilt, resentment, despair, and hopelessness.

Guideline5.3 The palliative care service facilitates religious, spiritual, and cultural rituals or practices as desired by patient and family, especially at and after the time of death.

\section{Domain 6: Cultural Aspects of Care.}

Guideline 6.1 The palliative care program serves each patient, family, and community in a culturally and linguistically appropriate manner. 
Guideline 6.2 The palliative care program strives to enhance its cultural and linguistic competence.

\section{Domain 7: Care of the Patient at the End of Life.}

Guideline 7.1 The interdisciplinary team identifies, communicates, and manages the signs and symptoms of patients at the end of life to meet the physical, psychological, spiritual, social, and cultural needs of the patients and families.

Guideline 7.2 The interdisciplinary team assesses and, in collaboration with the patient and family, develops, documents, and implements a care plan to address preventative and immediate treatment of actual or potential symptoms, patient and family preferences for site of care, attendance of family and/or community members at the bedside, and desire for other treatments and procedures.

Guideline 7.3 Respectful post-death care is delivered in a respectful manner that honors the patient and family culture and religious practices.

Guideline 7.4 An immediate bereavement plan is activated post-death.

\section{Domain 8: Ethical and Legal Aspects of Care.}

Guideline 8.1 The patient or surrogate's goals, preferences, and choices are respected within the limits of applicable state and federal law, current accepted standards of medical care, and professional standards of practice. Person-centered goals, preferences, and choices form the basis for plan of care.

Guideline 8.2 The palliative care program identifies, acknowledges, and addresses the complex ethical issues arising in the care of people with serious or lifethreatening illness. 
Guidelines 8.3 The provision of palliative care occurs in accordance with professional, state, and federal laws, regulations and current accepted standards of care (ClinicalPractice Guidelines for Quality Palliative Care, 2013). 


\section{Appendix B}

\section{Care and Communication Bundle}

(Nelson et al., 2005)

The nine measures are stratified by ICU length-of-stay triggers:

\section{ICU Day 1:}

1) Identify appropriate medical decision maker

2) Investigate advance directive status

3) Address cardiopulmonary resuscitation preference

4) Distribute information leaflet

5) Assess of pain at least every 4 hours

6) Appropriate pain management with alleviation of pain

\section{ICU Day 3:}

7) Offer social work support

8) Offer spiritual support

\section{ICU Day 5:}

9) Conduct an interdisciplinary family meeting 


\section{Appendix C}

\section{Palliative Care Service Consult Tool}

1. Would you be surprised if this patient were alive in one year? Score
a. $\quad$ Yes $=$ Score 3 points
b. No $=$ Score 0

2. Basic disease processes: Score 2 points each
a. Cancer (metastatic/recurrent)
b. Advanced COPD (on O2)
c. Neurological disease (difficulty swallowing or incontinent)
d. End-stage renal disease (considering stopping RRT)
e. Advanced CHF (one block DOE/repeated hospitalizations)
f. Not a candidate for curative surgery
g. Other terminal or incurable disease causing significant symptoms)
h. Greater than 3 hospitalizations, ED visits, ICU stays for incurable disease in past year

3. Uncontrolled symptoms or clinical conditions: Score 2 points each
a. Pain
g. Weight loss
b. Dyspnea
h. Constipation
c. Anxiety
i. Prolonged vent support
d. Depression
j. h/o cardiopulmonary arrest
e. Nausea
k. other 
f. Bowel obstruction

1. Total score

\section{Palliative Care Service Consult Tool}

\section{Anticipated functional status at time of discharge ECOG status (Eastern}

\section{Cooperative Oncology Group):}

\section{Grade Scale}

0-1 Fully active, able to carry on all pre-disease activities without restriction

2 Ambulatory and capable of most self-care, but unable to carry out any work activities. Up and about more than $50 \%$ of waking hours.

3 Capable of only limited self-care; confined to bed or chair more than $50 \%$ of waking hours or worse.

4 Completely disable. Cannot carry on any self-care. Totally confined to bed or chair.

\section{Psychological issues (patient or family): Score 2 points each}

a. Need to discuss end of life issues

b. Artificial nutrition or hydration

c. Need to evaluate for possible hospice referral

d. d. Unrealistic goals or expectations

Total Score:

Scoring Guidelines:

Total Score $\leq 8$ Problem directed; Consult if desired 
Total Score 9-11 Consider consult

Total Score $\geq 12$ strongly consider consult 
Appendix D

$\underline{\text { Patient Data Collection Sheet }}$

1. Patient Study Number:

2. Admitting Diagnosis:

3. Age:

4. Gender:

5. Length of Stay (on the MICU):

6. Past Medical History:

7. Palliative Care Service Consult Tool Score: 


\section{Appendix E}

\section{Care and Communication Bundle Data Collection}

\section{Patient Number:}

\section{Consult Tool Score:}

\section{Intensive Care Day \#1}

\section{Yes \\ No}

1) Identify appropriate medical decision maker

2) Investigate advance directive status

3) Address cardiopulmonary resuscitation preference

4) Distribute information leaflet

5) Pain assessment (is the patient being assessed for pain every four hours or more frequent, if needed)

6) Pain Management (is patient's pain being relieved)

\section{Intensive Care Day \#3}

7) Offer Social Work support

8) Offer Spiritual support 


\section{Intensive Care Day \#5}

Yes

No

9) Conduct an interdisciplinary family meeting

Total Score: (1 point for each 'yes' responses) 